\title{
Comparação do perfil epidemiológico entre indivíduos parasitados de Campo Mourão e Peabiru, Paraná
}

Comparison between epidemiological profile of parasitized individuals of Campo Mourão and Peabiru, Parana State

\section{Josiane Louback Motta}

Graduada em Farmácia pelo Centro Universitário Integrado.

\section{Suellen Fernanda Franco}

Graduada em Farmácia pelo Centro Universitário Integrado.

\section{Mariana Felgueira Pavanelli}

Doutora em Biociências e Fisiopatologia pela Universidade Estadual de Maringá. Docente do curso de farmácia do Centro Universitário Integrado.

\section{Resumo}

Objetivos: Comparar o perfil epidemiológico entre indivíduos parasitados das cidades de Campo Mourão e Peabiru, Paraná. Métodos: A pesquisa foi realizada em bancos de dados existentes em um laboratório de análises clínicas de cada município. Resultados: A taxa de infecção parasitária foi maior em Peabiru, com 17,9\% contra 2,9\% em Campo Mourão. Dentre os parasitos encontrados em ambos os municípios, a espécie em predominância foi Giardia intestinalis. Também foi possível observar que usuários do Sistema Único de Saúde, crianças e coletar três amostras de fezes aumentam a chance de diagnóstico de infecção parasitária. Conclusões: A população de Peabiru apresentou maior taxa de infecção parasitária e houve predomínio de infecção por protozoários. Alguns fatores parecem estar envolvidos na contaminação parasitária. Estudos epidemiológicos devem ser sempre realizados a fim de identificar infecções parasitárias e seus fatores associados e gerar informações úteis aos gestores municipais para melhora das condições de saneamento básico, contribuindo assim para a prevenção dos malefícios causados pelas enteroparasitoses.

Palavras-chave: Doenças Parasitárias; Epidemiologia; Fatores de Risco.

\section{Abstract}

The parasitic diseases intestinal are considered serious public health problem, accounting for high morbidity, due to poor sanitation conditions. Thus, the aim of this study was to compare the epidemiological profile of parasitized individuals in 
the cities of Campo Mourão and Peabiru, Paraná. The survey was conducted in existing databases in a clinical laboratory of each municipality. The parasitic infection rate was higher in Peabiru, with $17.9 \%$ against $2.9 \%$ in Campo Mourão. Among the parasites found in both municipalities, the species predominant was Giardia intestinalis. It was also observed that users of the Unified Health System, children and collect three samples of feces increase the chance of diagnosis of parasitic infection. We conclude that the population of Peabiru showed higher rates of parasitic infection and there was a

\section{Introdução}

As parasitoses intestinais constituem sério problema de saúde pública, embora apresentem baixa taxa de mortalidade, são responsáveis pelos elevados índices de morbidade, especialmente em países subdesenvolvidos ${ }^{1,2}$

No Brasil, a prevalência destas parasitoses é considerada bastante elevada em função das condições precárias de saneamento básico que favorecem a contaminação do solo, água e alimentos e das condições socioeconômicas da população. Neste sentido, as crianças podem ser as mais acometidas por essas infecções em razão dos hábitos de higiene não suficientemente consolidados e o sistema imunológico em desenvolvimento e para os adultos, a infecção geralmente está associada aos hábitos cotidianos de alimentação. ${ }^{1}$

A transmissão das enteroparasitoses ocorre por via oral-fecal, por meio da ingestão das formas infectantes dos parasitos presentes em água e predominance of infection by protozoa. Some factors seem to be involved in parasitic contamination. Epidemiological studies should always be performed in order to identify parasitic infections and their associated factors and generate useful information to municipal managers to improve the sanitation conditions, thus contributing to the prevention of harm caused by the intestinal parasites.

Keywords: Parasitic Diseases; Epidemiology; Risk Factors.

alimentos contaminados ${ }^{3,4}$ Após a infecção, o paciente pode ou não apresentar sintomas, estes quando surgem, correspondem principalmente a náuseas, vômito, diarreia, dores abdominais, diminuição do apetite, desnutrição e anemia, além do comprometimento no aprendizado e desenvolvimento físico e mental de crianças, e em casos mais graves o indivíduo pode até ir a óbito ${ }^{3,5,6}$

Os protozoários intestinais mais frequentemente encontrados em seres humanos são Entamoeba coli, Entamoeba histolytica, Endolimax nana e Giardia intestinalis. Dentre os helmintos destacam-se: Ascaris lumbricoides, Enterobius vermiculares, Trichuris trichiura, Schistosoma mansoni, Strongyloides stercoralis e Ancylostoma duodenale. ${ }^{3,7}$

O diagnóstico dessas infecções é realizado por meio dos exames parasitológicos de fezes, sendo as metodologias mais utilizadas as técnicas de Hoffmann et al ${ }^{8}$.; para a pesquisa de ovos e, para a identificação de cistos as técnicas 
utilizadas são as de Faust et al $^{9}$. e Ritchie ${ }^{10}$ e para pesquisa de larvas, opta-se pela técnica de Baermann-Moraes ${ }^{11,12,13}$

O tratamento antiparasitário varia conforme a classe do parasito. Para as infecções causadas por helmintos, os fármacos mais utilizados são albenzadol, mebendazol, tiabendazol, praziquantel e ivermectina. Já para os protozoários os medicamentos de escolha são nitazoxanida, metronidazol, tinidazol e secnidazol. ${ }^{14,15}$ Entretanto a terapia medicamentosa visa eliminar o parasito do indivíduo infectado, porém é necessário adotar medidas de controle que sejam capazes de erradicar as infecções parasitárias de determinada localidade.

Neste sentido, as atividades de educação em saúde são consideradas importantes estratégias no controle e redução do número de parasitoses. O processo educativo visa promover ações curativas e profiláticas que podem ser realizadas por diferentes profissionais de saúde . $^{16,17}$

Considerando que as enteroparasitoses podem causar sérios danos à saúde de crianças e adultos, sendo consideradas importantes indicadores epidemiológicos que contribuem para a construção de políticas públicas de promoção à saúde e melhoria da qualidade de vida da população e que até o presente momento não há estudos epidemiológicos comparativos sobre a prevalência de enteroparasitos nas cidades de Campo Mourão e Peabiru, PR, o objetivo deste trabalho foi comparar o perfil epidemiológico entre indivíduos parasitados das cidades de Campo Mourão e Peabiru, Paraná.

\section{Métodos}

O município de Campo Mourão possui 92.930 habitantes e sete laboratórios de análises clínicas que atendem convênios particulares e o Sistema Único de Saúde (SUS), e também um laboratório pertencente ao Consórcio Intermunicipal de Saúde dos Municípios da COMCAM (CISCOMCAM), que atende somente o SUS. A cidade de Peabiru possui 14.144 habitantes e dois laboratórios de análises clínicas. Ambos os municípios ficam localizados no noroeste do Estado, na mesorregião centro-ocidental paranaense. ${ }^{18}$

Foi escolhido um laboratório de análises clínicas com atendimento ao sistema público e privado de saúde de cada município, por conveniência, para a execução deste estudo. Assim, foi realizada uma pesquisa epidemiológica em bancos de dados de cada um dos laboratórios escolhidos. Nessa pesquisa foram coletados dados de indivíduos que realizaram exames parasitológicos de fezes durante um ano. As variáveis analisadas foram idade, gênero, quantidades de amostras de fezes coletadas, tipo de convênio médico e o resultado do exame parasitológico. Os dados foram utilizados para comparação do perfil de infecções parasitárias existentes nos dois municípios pesquisados. 
Os dados foram analisados por meio de cálculo de Razão de Prevalência utilizando o software livre Epi Info. Foi considerado nível de significância superior a 95\%.

Este estudo seguiu os princípios éticos contidos nas Resoluções 466/12 e 510/2016 do Conselho Nacional Conselho de Saúde e foi aprovado pelo Comitê de Ética para Pesquisa Envolvendo Seres Humanos do Centro Universitário Integrado sob o Certificado de Apresentação p a r a A p r e c i a çã o É ti c a n o 46799015.4.0000.0092.

\section{Resultados e discussão}

Foram analisados 629 pacientes do município de Campo Mourão, com média de idade de 24,5 $\pm 21,2$ anos e $56 \%$ pertencente ao gênero feminino. Em Peabiru, foram coletados dados de 791 pacientes, dos quais a média de idade foi de $21,4 \pm 20,9$ anos e a prevalência do gênero feminino foi de $57,4 \%$.

No estudo de Costa et al. ${ }^{3}$ foi observado que a quantidade de pedidos de exames parasitológicos de fezes nas consultas médicas realizadas foi baixa, e mesmo quando estes foram requeridos, houve um alto índice de pessoas que não retornaram às consultas para informe do diagnóstico. Esse fato pode também ter ocorrido em Campo Mourão, pois a quantidade de exames de fezes realizados foi baixa quando comparado com Peabiru, que representa $15,2 \%$ da população de Campo Mourão. ${ }^{18}$
A taxa de infecção parasitária em Peabiru foi de 17,9\%, contra 2,9\% em Campo Mourão, este resultado corresponde a $0,02 \%$ da população total de Campo Mourão e 1\% de Peabiru. Segundo dados do IBGE Cidades ${ }^{18}$, a taxa de óbitos oriundos de doenças infecciosas e parasitárias no município de Campo Mourão foi de $0,04 \%$ e para Peabiru $0,07 \%$. Comparando estes números com os resultados do estudo, nota-se que a elevada prevalência de parasitos pode estar relacionada ao maior índice de óbitos registrado. A prevalência de cada parasito encontrado está descrita na Tabela 1.

A alta prevalência de parasitoses intestinais em Peabiru pode estar associada à infraestrutura do município, pois trata-se de uma cidade de pequeno porte (14.144 habitantes) e predomínio de atividade agrícola. ${ }^{18}$ Borges et al. ${ }^{19}$, observaram que a ocorrência de helmintos e protozoários em pacientes atendidos no Hospital Municipal de Cumari (GO) foi de $36,2 \%$, sendo $33,6 \%$ de casos de $G$. intestinalis, e associou que a elevada incidência deste protozoário pode estar relacionada à economia local, uma vez que os solos das fazendas podem estar contaminados com fezes humanas em razão das defecações em locais inapropriados, o que facilita a transmissão fecal-oral.

A presença de enteroparasitoses de acordo com alguns autores também pode estar relacionada a locais com deficiência de saneamento básico, excesso de moradores em uma residência, poucos cômodos, ao consumo de água oriunda de poços e hábitos de lazer como nadar em rios, 
uma vez que a água pode estar contaminada com formas parasitárias infectantes ${ }^{19,20}$.

Dentre os protozoários encontrados nos dois municípios deste estudo, a espécie que predominou foi $G$. intestinalis, corroborando com os estudos de Borges et al. ${ }^{19}$ no estado de Goiás e Silva et al. ${ }^{21}$ no Maranhão. Outros autores também encontraram maior prevalência de $G$. intestinalis, nos estados do Paraná, ${ }^{13,22,23}$ São Paulo $^{24,25}$ Minas Gerais ${ }^{26}$ e Bahia ${ }^{27}$.

A elevada prevalência de $G$. intestinalis pode estar associada ao contato com o solo contaminado, falta de saneamento básico, ingestão de água não tratada e alimentos lavados de maneira inadequada, os quais tornam-se veículos para a transmissão de parasitos. $^{28}$ A giardíase, infecção por G. intestinalis, é mais frequente em crianças em idade escolar em função dos hábitos de higiene e contato direto com outras crianças. ${ }^{29,30}$

Diferenças nas taxas de infeç̧ão entre os dois municípios estudados foram observadas para $E$. coli, E. nana, E. vermicularis, T. trichiura, S. stercoralis e Taenia sp. E. coli foi encontrada nos dois municípios estudados e $E$. nana foi encontrada apenas em Peabiru. Miranda et al. ${ }^{31}$, verificaram maior prevalência desses dois comensais, $56 \%$ de $E$. coli e $35,1 \%$ E. nana e dentre os protozoários patogênicos, o mais frequente foi G. intestinalis com $30,2 \%$ de amostras positivas.

A baixa ocorrência de $E$. nana, encontrado apenas em Peabiru, pode ser explicada pelo fato do município ter poucos habitantes, já que este parasito apresenta maior prevalência em lugares com grandes aglomerações de pessoas, uma vez que a expansão populacional favorece sua transmissão entre humanos. ${ }^{19}$ Além disso, pode-se associar a baixa prevalência ao possível uso de técnicas parasitológicas menos sensíveis para a detecção deste protozoário, que apresenta tamanho reduzido. Apesar das técnicas rotineiras de laboratório serem de fácil execução, a falta de treinamento dos analistas dificulta o diagnóstico laboratorial deste protozoário. $^{32}$

Dentre os helmintos, verificou-se maior prevalência em Campo Mourão, com 11,1\% de infecções por Taenia sp. (não diagnosticada em Peabiru) e 5,6\% de infecções por Ascaris lumbricoides, contra 0,6\% em Peabiru. Os demais helmintos evidenciados ( $E$. vermicularis, T. trichiura e S. stercoralis) foram isolados apenas em Peabiru.

A alta prevalência de Taenia sp. pode estar relacionada a hábitos alimentares ou socioculturais. ${ }^{33} 0$ contato próximo entre humanos e suínos favorece a disseminação da infecção por Taenia sp. e também ressalta que esta parasitose apresenta baixa prevalência em crianças. ${ }^{24}$ A teníase é a doença causada pela forma adulta de Taenia solium ou saginata, a infecção ocorre por meio da ingestão de carne crua ou mal cozida de bovinos ou suínos contendo cisticercos ${ }^{3,34}$

Como medidas eficazes para o controle da 
teníase aplica-se a inspeção higiênico-sanitária das carnes e tornar obrigatório o registro de casos positivos na região, estes dados devem ficar disponíveis aos produtores possibilitando o monitoramento de cada abate..$^{35}$ Ainda, segundo este autor, a infecção em bovinos de corte por esta doença pode estar associada ao fato de que na maioria das vezes esses animais são criados e engordados na própria propriedade, o que facilita a transmissão da doença.

O helminto $E$. vermicularis, encontrado apenas em Peabiru, não é frequentemente diagnosticado nos métodos parasitológicos habitualmente realizados. Miranda et al. ${ }^{31}$, encontraram baixa prevalência deste parasito em 4,6\% das amostras positivas, pois utilizaram apenas a técnica de Hoffmann et al. ${ }^{8}$ para identificação das enteroparasitoses em seu estudo e de acordo com os autores, esse parasito exige um método de diagnóstico específico: a técnica da fita gomada ${ }^{36}$. Por esse motivo não é possível afirmar conclusivamente que houve baixa prevalência deste parasito na população estudada, corroborando com Cardoso et al. ${ }^{37}$ e Silva et al. ${ }^{21}$

A baixa ocorrência de $S$. stercoralis também pode estar relacionada ao emprego de métodos diagnósticos menos específicos. $O$ índice de infecção por esse helminto pode ter sido subestimado, devido a não utilização da técnica de Baermann-Moraes ${ }^{11}$ que é a mais indicada para o diagnóstico parasitológico de larvas ${ }^{4,38}$

Foram encontradas infecções parasitárias mistas apenas em Peabiru, com prevalência de
4,93\% de associação entre $G$. intestinalis e $E$. coli e 2,82\% entre $G$. intestinalis e E. nana. Nos estudos de Borges et al. ${ }^{19}$ e Bica et al. ${ }^{39}$, também foi encontrada maior associação de $G$. intestinalis e E. coli. A elevada prevalência de poliparasitados pode estar associada à falta de saneamento básico, condições de higiene e elevado número de moradores na mesma residência, no entanto, esses fatores não foram investigados neste estudo ${ }^{26}$.

As infecções parasitárias evidenciadas nos dois municípios deste estudo foram relacionadas à algumas variáveis para determinação de medidas epidemiológicas de associação e efeito (Tabela 2). Não foi encontrada associação significativa para gênero, já para faixa etária, em ambos os municípios foi observada maior frequência de parasitos nas crianças com idade entre 0 e 15 anos, corroborando com Borges et al. ${ }^{19}$ e Nascimento ${ }^{40}$. Este resultado pode estar associado aos hábitos de higiene não consolidados, educação sanitária, ambiente escolar, ao ato de brincar e possuir animais de estimação. ${ }^{1,23,28}$ Comparando crianças (0 a 15 anos) e adultos, apenas foi evidenciada associação significativa em Campo Mourão, com Razão de Prevalência (RP) de 3,96, Intervalo de Confiança (IC) de 1,32 a 11,91 e pvalor $<0,01$.

Em Peabiru, quando comparadas crianças de 0 a 4 anos e de 5 a 10 anos, observou-se que há 2,1 mais chances de infecção parasitária na faixa etária de 5 a 10 anos (IC: 1,3 a 3,4 e p-valor<0,01). A elevada prevalência de enteroparasitos em crianças de 
maior idade pode estar associada à independência de cuidados higiênicos alheios e contato com o meio ambiente, fatores estes que podem facilitar a contaminação por parasitos intestinais ${ }^{30}$ As crianças são as mais acometidas pelas infecções parasitárias em função dos princípios básicos de higiene ainda em consolidação e em relação aos adolescentes, estes pouco se preocupam com os hábitos de higiene ${ }^{28}$.

Em relação ao tipo de convênio, 39,7\% dos indivíduos de Campo Mourão utilizavam o SUS, contra $87,5 \%$ dos pacientes atendidos em Peabiru. A baixa quantidade de pessoas que utilizaram o SUS em Campo Mourão pode ser explicada pelo fato do município possuir o laboratório CISCOMCAM, que possui atendimento direcionado exclusivamente aos pacientes do sistema público de saúde. Entretanto, somente para o município de Campo Mourão foi encontrada associação significativa entre infecção parasitária e ser usuário do SUS (RP 3,94, IC: 1,42 a 10,92 e p-valor<0,01).

Há uma tendência de que aqueles que são usuários do SUS possam estar mais expostos às infecções parasitárias em função das condições socioeconômicas. As parasitoses intestinais são mais frequentes em famílias de nível social mais baixo e com menor grau de escolaridade e este número diminui gradativamente à medida em que aumentam os níveis de conhecimento educacional e renda familiar. $1,2,6,31$

Quanto à quantidade de amostras fecais coletadas, dos pacientes de Campo Mourão apenas $13 \%$ coletaram três amostras, sem associação significativa com a detecção de parasitos. Já em Peabiru, 30,3\% dos indivíduos coletaram três amostras e este fator aumentou em 2,4 vezes a chance de diagnóstico positivo (IC: 1,81 a 3,25, p-valor $<0,01$ ). A maior quantidade de pessoas que coletaram três amostras de fezes observada em Peabiru pode também explicar a maior taxa de infecção parasitária apresentada pelo município.

A coleta de mais de uma amostra de fezes e a associação de dois ou mais métodos de diagnóstico aumentam a sensibilidade de detecção dos parasitos. ${ }^{12,21,29}$ Belloto et al. ${ }^{24}$, observaram baixa ocorrência de doenças parasitárias em escolares da rede pública de Mirassol, São Paulo, e associaram o resultado ao fato de ter obtido apenas uma amostra de fezes de cada criança para a realização dos exames parasitológicos, já que há uma década, uma pesquisa realizada na mesma escola encontrou prevalência de $63,9 \%$ de enteroparasitos.

Este estudo possibilitou conhecer o perfil epidemiológico do portador de enteroparasitos de Peabiru e Campo Mourão e relacionar as características epidemiológicas às infecções parasitárias para conhecer os fatores de risco associados à doença.

\section{Considerações finais}

Pode-se concluir que a população de Peabiru apresentou maior taxa de infecção parasitária do que a de Campo Mourão e a espécie em 
predominância nos dois municípios foi $G$. intestinalis. Também foi possível evidenciar que o tipo de convênio, idade e quantidade de amostras coletadas estão associadas à presença de infecção parasitária.

\section{Embora a taxa de infecção parasitária} encontrada em Campo Mourão foi relativamente baixa, estudos epidemiológicos como este devem ser sempre realizados a fim de identificar infecções parasitárias e seus fatores associados e gerar informações úteis aos gestores municipais para a melhora das condições de saneamento básico, contribuindo assim para a prevenção dos malefícios causados pelas enteroparasitoses. Deve ser incentivada a realização de campanhas educativas sobre hábitos saudáveis de higiene e a importância da correta lavagem de mãos e dos alimentos, visando à erradicação das parasitoses intestinais e melhoria da qualidade de vida da população.

\section{Referências}

1.Almeida IA. Prevalência de enteroparasitoses em crianças hospitalizadas em Pelotas, Rio Grande Do Sul, Brasil. [Dissertação] Pelotas: Programa de Pós-Graduação em Parasitologia, Universidade Federal de Pelotas; 2013.68f.

2. Ferraz RRN, Barnabé AS, Porcy C, Junior AD, Feitosa T, Figueiredo PM. Parasitoses Intestinais e baixo índice de Gini em Macapá-AP e Timon-MA, Brasil. Caderno Saúde Coletiva.Rio de Janeiro. 2014; 22(2):173-176.

3. Costa ACN, Borges BC, Costa AV, Ramos MF, Gomes JM, Gomes JM, et al. Levantamento de acometidos por enteroparasitoses de acordo com a idade e sexo e sua relação com o meio onde está inserido o psf Prado da cidade de Paracatu-MG. Revista de Patologia Tropical. 2012 abr-jun; 41(2):203-214.

4. Gomes PDMF, Nunes VLB, Knechtel DS, Brilhante AF. Enteroparasitoses em escolares do Distrito Águas do Miranda, Município de Bonito, Mato Grosso do Sul. Revista de Patologia Tropical. 2010 out-dez;39(4):299-307.

${ }^{5}$.Addum FM, Serra CG, Sessa KS, Izoton LM, Santos TB. Planejamento local, saúde ambiental e Estratégia Saúde da Família: uma análise do uso de ferramentas de gestão para a redução do risco de contaminação de enteroparasitoses no município de Venda Nova do Imigrante. Revista de Saúde Coletiva. 2011; 21(3):955-977.

6. Vasconcelos IAB, Oliveira JW, Cabral FRF, Coutinho HDM, Menezes IRA. Prevalência de parasitoses intestinais entre crianças de 4-12 anos de Grato, Estado do Ceará: um problema recorrente de saúde pública. Revista Acta Scientiaurum Health Science.Maringá. 2011;33(1):35-41.

7. Seger J, Souza WM, Marangoni JCF, Maschio VJ, Chielli EO. Prevalência de parasitas intestinais na população do Bairro Salete, município de São Miguel do Oeste, SC. Revista UNOESC \& Ciência-ACBS.2010 jan-jun;1(1):53-56.

${ }^{8}$. Hoffmann WA, Pons JA, Janer JL. The sedimentation concentration method in schistosomiasis mansoni. Puerto Rico J. Public. Health.1934; 9:283-291.

9. Faust EC, Sawitz W, Tobie J, Odom V, Lincicome CPEDR. Comparative efficiency of various technics for the diagnosis of protozoa and helminths in feces. Journal of Parasitology.1939; 25(3):241-262.

10. Ritchie LS. An ether sedimentation technique for routhine stool examinations. Bull U S Army Med Dep. $1948 ; 4(8): 326$.

11. Moraes RG. Contribuição para o estudo do Strongyloides stercoralis e da estrongiloidose no Brasil. Revista do Serviço Especial de Saúde Pública.1948;1(3):507-624.

12. Menezes RAO, Gomes MSM, Barbosa FHF, Machado RLD, Andrade RF, Couto AARDEA. Sensibilidade de métodos parasitológicos para o diagnóstico das enteroparasitoses em Macapá-Amapá, Brasil. Revista de Biologia e Ciências da Terra.2013;13(2):63-73.

13. Lopes CR, Salamaia FH, Molinari LM. Diferentes parasitos intestinais em crianças de um a dez anos atendidas em um laboratório de análises clinícas na cidade de Marialva, Paraná, Brasil. Revista Saúde e Pesquisa. 2012 maio-ago; 5(2):290297. 
${ }^{14}$.Braz AS, Andrade CAF, Mota LMH, Lima CMBL. Recomendações da Sociedade Brasileira de Reumatologia sobre diagnóstico e tratamento das parasitoses intestinais em pacientes com doenças reumáticas autoimunes. Revista Brasileira de Reumatologia. 2015;55(1):1-1.

15. Andrade EC, Leite ICG, Rodrigues VO, Cesca MG. Parasitoses Intestinais: uma revisão sobre seus aspectos sociais, epidemiológicos, clínicos terapêuticos. Revista de Atenção Primária à Saúde. Juiz de Fora. 2010 abr-jun;13(2):231-240.

16. Almeida RA, Pequeno GA, Amorim FDB, Amador DD, Marinho AH, Rosalmeida MDP, et al. Jogo parasitológico: uma estratégia no ensino-aprendizagem da parasitologia. Revista Rede de Cuidados em Saúde. 2012; 6(1):1-1

17. Miranda SVC. Atuação dos profissionais da estratégia saúde da família (ESF) frente às principais parasitoses intestinais. [Trabalho de Conclusão de Curso] Corinto: Universidade Federal de Minas Gerais; 2013.65f.

18. Instituto brasileiro de Geografia e Estatística: IBGE Cidades. [Acesso em 04 de outubro de 2015]. Disponível em: http://www.cidades.ibge.gov.br/xtras/home.php .

19. Borges WF, Marciano FM, Oliveira HB. Parasitos intestinais: elevada prevalência de Giardia lamblia em pacientes atendidos pelo serviço público de saúde da região sudeste de Goiás, Brasil. Revista de Patologia Tropical. 2011 abr-jun; 40(2):149-157.

${ }^{20}$. Fonseca EOL, Teixeira MG, Barreto ML, Carmo EH, Costa MDCN. Prevalência e fatores associados às geo-helmintíases em crianças residentes em municípios com baixo IDH no Norte e Nordeste brasileiros. Cadernos de Saúde Pública. 2010 jan; 26(1):143-152.

21. Silva FS, Paulo ADDAC, Braga CMM, Almeida RJ, Galvao VP. Frequência de Parasitos intestinais no município de chapadinha, Maranhão, Brasil. Revista de Patologia tropical. 2010 jan-mar; 39(1):63-68.

22. Netto AM. Infecções por protozoários e seus fatores de riscos em escolares do Município de Peabiru, Pr. [Trabalho de Conclusão de Curso] Campo Mourão: Graduação em Farmácia, Faculdade Integrado de Campo Mourão; $2012.13 f$.

${ }^{23}$. Rodrigues R, Hirano MM, Larentes TS. Verificação do parasitismo em centros de educação infantil de Paranavai (PR): Envolvendo ações sanitárias primárias desenvolvidas nessa comunidade. Revista Saúde e Pesquisa. 2014 set-dez; 7(3):409-421.

${ }^{24}$. Belloto MVT, Santos Junior JE, Macedo EA, Ponce A, Galisteu KJ, Castro Ede, et al. Enteroparasitoses numa população de escolares da rede pública de ensino do Município de Mirassol. Revista Pan-Amazônica de Saúde. São Paulo, 2011; 2(1):37-44.

${ }^{25}$. Lodo M, Oliveira CGB, Fonseca ALA, Caputto LZ, Packer MLT, Valenti VE, et al. Prevalência de enteroparasitas em município do interior paulista. Revista Brasileira de Crescimento e Desenvolvimento Humano. 2010 set; 20(3):769- 777.

26. Andrade EC, Leite ICG, Vieira MTde, Abramo C, Tibiriçá SHC, Silva PL. Prevalencia de parasitoses intestinais em comunidade quilombola no município de Bias Fortes, Estado de Minas Gerais, Brasil, 2008. Revista Epidemiologia e Serviços de Saúde. 2011 jul-set;20(8):337-344.

27. Ferreira VS, Lima AG, Pessoa CS, Paz FSS, Jesus Jde. Estudo comparativo das enteroparasitoses ocorrentes em duas áreas de Barreiras, Bahia.Natureza on line. 2013; 11(2):90-95.

${ }^{28}$. Ludwig KM, Ribeiro ALT, Conte AdeC, Decleva DV, Ribeiro JTD. Ocorrência de enteroparasitoses na população de um bairro da cidade de Cândido Mota-SP. Journal of the Healht Sciences Institute. 2012; 30(3):271-276.

29. Genuino IP. Ocorrência de enteroparasitos em manipuladores de alimentos das cantinas da Universidade Federal da Paraíba/ Campus I. [Trabalho de Conclusão de Curso] João Pessoa: Graduação em Farmácia, Universidade Federal da Paraíba; 2014.54f.

30. Pedraza DF, Queiroz Dde, Sales MC. Doenças infecciosas em crianças pré-escolares brasileiras assistidas em creches. Revista Ciência \& Saúde Coletiva. 2014; 19(2):511-528.

31. Miranda GC, Dattoli VCC, Lima AD. Enteroparasitos e condições socioeconômicas e sanitárias em uma comunidade quilombola do semiárido baiano. Revista de Patologia Tropical. 2010 jan-mar; 39(1):48-55.

${ }^{32}$.Eymael D, Schuh GM, Tavares RG. Padronização do diagnóstico de Blastocystis hominis por diferentes técnicas de coloração. Revista da Sociedade Brasileira de Medicina Tropical. 2010 maio-jun; 43(3):309-312.

33. Afonso MBVdeO. Prevalência de Taenia saginata/Cysticercus bovis na Região Autónoma da Madeira. [Dissertação de Mestrado]. Lisboa. Faculdade de Medicina Veterinária, 2008. 102f.

${ }^{34}$. Coeli GNM, Tiengo RR, Silva ACDe, Fernandes JOM, Silva GC, Silva LUM. Neurocisticercose nodular calcificada com sinais de reativação. Radiologia Brasileira. 2012 set-out; 45(5):291-293. 
${ }^{35}$. Rossi GAM, Grisolio APR, Prata LF, Burger KP, Hoppe EGL. Situação da cisticercose bovina no Brasil. Revista Semina Ciências Agrárias. 2014 mar-abr; 35(1):927-938.

${ }^{36}$. Graham DF. A device for the diagnostic on the Enterobius vermicularis infection. American Journal of Tropical Medicine. 1941;21(1):159-161.

37. Cardoso FDP, Araujo BM, Batista HL,Galvao WG. Prevalência de enteroparasitoses em escolares de 06 a 14 anos do município de Araguaiana-Tocantins. Revista Eletrônica de Farmácia.2010; 7(1):54-64.

${ }^{38}$. Visser S, Giatti LL, Carvalho RACde, Guerreiro JCH. Estudo da associação entre fatores socioambientais e prevalência de parasitose intestinal em área periférica da cidade de Manaus (AM, Brasil). Revista Ciência \& Saúde Coletiva. 2011; 16(8):3481-3492.

39. Bica VC, Dillenburg AF,Tasca T. Diagnóstico laboratorial da giardiose humana: Comparação entre as técnicas de sedimentação espontânea em água e de centrífugo-flutuação em solução de sulfato de zinco. Revista do Hospital de Clinicas de Porto Alegre. 2011;31(1):39-45.

40. Nascimento YM. Estudo acerca da ocorrência de helmintos intestinais em dois municípios do estado do Paraíba. [Trabalho de Conclusão de Curso] João Pessoa: Graduação em Farmácia, Universidade Federal da Paraíba; $2014.65 f$.

\section{Tabelas}

Tabela 1. Prevalência de cada parasito nos municípios de Campo Mourão e Peabiru, Paraná.

\begin{tabular}{|l|c|c|}
\hline \multirow{2}{*}{\multicolumn{2}{|c|}{ Parasito }} & \multicolumn{2}{|c|}{ Prevalência (\%) } \\
\cline { 2 - 3 } & Campo Mourão & Peabiru \\
\hline Giardia intestinalis & 72,2 & 73,1 \\
\hline Entamoeba coli & 11,2 & 14,1 \\
\hline Endolimax nana & 0,0 & 8,3 \\
\hline Enterobius vermicularis & 0,0 & 2,6 \\
\hline Taenia sp. & 11,1 & 0,0 \\
\hline Ascaris lumbricóides & 5,6 & 0,6 \\
\hline Trichuris trichiura & 0,0 & 0,6 \\
\hline Strongyloides stercoralis & 0,0 & 0,6 \\
\hline
\end{tabular}


Tabela 2. Distribuição de aspectos epidemiológicos em indivíduos parasitados nos municípios de Campo Mourão e Peabiru, Paraná.

\begin{tabular}{|c|c|c|c|c|}
\hline \multirow{2}{*}{$\begin{array}{l}\text { Aspectos } \\
\text { epidemiológicos }\end{array}$} & \multicolumn{2}{|c|}{ Campo Mourão } & \multicolumn{2}{|c|}{ Peabiru } \\
\hline & $\mathbf{n}$ & $\%$ & $\mathbf{n}$ & $\%$ \\
\hline \multicolumn{5}{|l|}{ Convênio } \\
\hline SUS & $13^{*}$ & 72,2 & 125 & 62,0 \\
\hline Particular & 5 & 27,8 & 17 & 12,0 \\
\hline \multicolumn{5}{|c|}{$\begin{array}{l}\text { Quantidade de amostras } \\
\text { coletadas }\end{array}$} \\
\hline 1 amostra de fezes & 16 & 88,9 & 69 & 48,6 \\
\hline 3 amostras de fezes & 2 & 11,1 & $73^{*}$ & 51,4 \\
\hline \multicolumn{5}{|l|}{ Gênero } \\
\hline Feminino & 10 & 55,6 & 86 & 60,6 \\
\hline Masculino & 8 & 44,4 & 56 & 39,4 \\
\hline \multicolumn{5}{|l|}{ Idade (anos) } \\
\hline 0 a 15 & $14 *$ & 77,8 & 83 & 58,5 \\
\hline 16 a 40 & 2 & 11,1 & 31 & 21,8 \\
\hline 41 a 60 & 2 & 11,1 & 17 & 12,0 \\
\hline$>60$ & 0 & 0,0 & 11 & 7,7 \\
\hline Total & 18 & 100 & 142 & 100 \\
\hline
\end{tabular}

*Representa associação significativa com infecção parasitária $(p<0,05)$. 